\title{
Analysis of the Plantar Pressure Characteristics of Action Flying Kick for Martial Arts Athletes
}

\author{
Jin Fei Tan*
}

Sport and Art College of Hunan Agricultural University, Changsha 410128, Hunan, China

\begin{abstract}
Martial arts as the quintessence of the Chinese nation, after a thousand years of forging, its contents are rich and varied, the performance of a variety of forms, the value of its potential is enormous. This paper, regarding the action flying kick the as a starting point in competitive of martial arts, analysis the plantar pressure characteristics of action flying kick for martial arts athletes, and provided some quantization theory for the flying feet of jumping movements, give some suggestions in the process to jumping which may be injured. Firstly, this paper discussed the methods and steps for the analysis of the plantar pressure characteristics of action flying kick for martial arts athletes, summing up the process of the plantar pressure analysis and the experimental process. Then, the paper given six Jilin province martial arts athletes flying kick foot jump for bottom pressure test of the experimental results, and analyzed the experimental data in detail. By analyzing the experimental results, it can reveal regular pattern of the diversification of plantar pressure, so as to provide reference data for improving the effect of training and preventing injuries.
\end{abstract}

Keywords: Action flying kick, martial arts, plantar pressure characteristics.

\section{INTRODUCTION}

Martial arts as the quintessence of the Chinese nation, after a thousand years of forging, its contents are rich and varied, the performance of a variety of forms, the value of its potential is enormous. With the constant improvement of the level of martial arts competitions in recent years, and continuously enhance the strength of competition, the introduction of modern technology to a quantitative analysis of athletes is very necessary.

Plantar pressure analysis is an emerging research tools which has been used in many field, such as sports biomechanics, orthopedics, rehabilitation medicine, footwear and so on. Plantar pressure analysis not only allows us to have a more in-depth understanding of the normal gait; also provide a reference for the analysis of competitive sports, public health; morbid foot plantar pressure analysis provides a baseline of normal standards. So martial arts can also use this new method to make some quantitative research. However, the plantar pressure study of martial arts, there is few, and the study of jumping and flying action of martial arts is little. Therefore, the Analysis of the plantar pressure characteristics of action flying kick for martial arts athletes is very necessary.

In 1882 [1], Beely took the lead on the plantar pressure more scientific research. Real systematically plantar pressure analysis is used in the sport biomechanics and began in the $1950 \mathrm{~s}$, in the past twenty or thirty years has developed rapidly, has from pure theory to sports biomechanical scholars, doctors, sports equipment designers hand tools. At present,

*Address correspondence to this author at the Sport and Art College of Hunan Agricultural University, Changsha 410128, Hunan, China;

Tel: 18932432557; E-mail: thepsb01@163.com the plantar pressure analysis has entered the movement, the computer precise quantitative analysis of $3 \mathrm{~d}$ simulation of the stage [2]. In 1997, the 16th international conference on biomechanics, the gait research papers has about a third of the total number of papers. In recent years, Gait Posture [3], Ergonomics [4], Clinical Biomechanics [5], Journal of Biomechanics [6] and other international academic journals published a large number of gait research papers, most of these papers relating to the plantar pressure distribution and measurement analysis.

Analysis of plantar pressure in developed countries started early, and accumulated a large number of parameter values. International famous plantar pressure analyzer are established on the basis of large sample parameter values. They have worked focuses on the use of the analyzer to indepth and meticulous research plantar pressure. Stebbins, measured the footprint of 12 normal children, and results showed that the pressure might be a more appropriate measure of stress values. This presents a challenge long-term use pressure as a common evaluation parameters. Morag, collected 55 healthy people walking the plantar peak pressure and eight other structural and functional data, established a prediction model of the plantar peak pressure. Establish forecast model of plantar pressure can be observed, to identify potential pathogenic factors, to help reduce the injured athletes, plantar pressure of high risk patients. Mary Josephine and Hessertused the plantar pressure to test the insole, test the nine young people and six old people walking on the run, the test parameters including a complete gait phase characteristics, including average, maximum pressure and the pressure value. At present, the application of plantar pressure test system are mostly concentrated in the clinical medicine, sports, public health, and a quantitative analysis of competitive martial arts research is less. This paper, regarding the action flying kick the as a starting point in 
Table 1. The basic information registration sheet of research object.

\begin{tabular}{|c|c|c|c|c|c|c|}
\hline 1 & Male & 17 & 165 & 50 & 22 & Stage 2 \\
\hline 3 & Male & 18 & 170 & 55 & 23 & Stage 2 \\
\hline 6 & Male & 19 & 174 & 60 & 25 & Stage 2 \\
\hline
\end{tabular}

competitive of martial arts, analysis the plantar pressure characteristics of action flying kick for martial arts athletes, and provided some quantization theory for the flying feet of jumping movements, give some suggestions in the process to jumping which may be injured (abstract).

\section{OVERVIEW OF PLANTAR PRESSURE ANALYSIS AND ACTION FLYING KICK}

According to plantar pressure measurement and analysis of the development and use of technology can be divided into footprint method, foot pressure scanner, direct visualization techniques, pressure plate and plantar pressure insoles and others. Various techniques have advantages and disadvantages, so the two or more methods together organically in order to obtain satisfactory results. Currently, the measurement of dynamic plantar pressure and static, barefoot and wearing shoes two scenarios. Parameters assessed are: pressure, peak and mean stresses, the contact area, the stress time curve, single feet standing time, plantar pressure center track, and the gravity analysis [7].

In recent years, the United States Tekscan's F-scan system, Belgium Footscan tablet system and insole systems, Switzerland Kistler force platform, Germany Novel Emed Tablet systems and Pedar insole system for more in-depth research. Especially the two former applications are more widely.

The action name flying kick in martial arts routine, in 1958, martial arts organizations by the state physical culture and sports of the routine training, the traditional flying feet was developed to flying kick $[8,9]$, in the traditional routine, chaquan, huaquan, paoquan, and hongquan and so on all has this action, the original named fly feet, jump flying kick, this movement exists when a martial arts, then through the training and development, provisions must be made in the empty action, after been smacked in the air to the ground, to complete the action. Flying kick movement can be divided into four stages, namely: run, jump, fly, landing four stages [1012]. To short run-up obtained after the motive power of brake, jumping to horizontal velocity into vertical speed, jumping to complete. Flight movement to be smacked in the air flying kick, in which the forward hand struck the foot of the trunk, feet flying legs straight, the other leg hip, bend your knees, attached to the flying feet and legs. Last completed action is land on the ground.

\section{ANALYSIS METHOD AND STEPS}

\subsection{The Research Object}

This study selected six excellent martial arts athlete in Jilin province martial arts team as the research object, basic conditions are shown in Table 1. Six players were trained in long-term system, movement level above level. Through foot history and physical examination to determine the object of study, no foot illness and injury, foot anatomy and physiology all normal, athletic ability is good.

\subsection{The Analysis Method and Testing Steps}

In the process of analysis of the plantar pressure characteristics of action flying kick for martial arts athletes, it should use a lot of research methods, in the first place, by looking up the literature about the flying kick, understand the basic situation of flying kick, and consulting relevant experts and coaches, to flying kick behavioral essentials, technical features in-depth understanding, then carries on the experiment using pressure test system, get the corresponding data, finally, to process the data collection, input data into EXCEL data sorting, finishing SPSS11.7 complete data input for the data analysis [13-15], calculate the standard deviation and average, according to the experimental results, in the experiment, and discussed the results found that the problem analysis, through discussion is more objective and more reliable experimental data are obtained. The whole process of analysis of the plantar pressure characteristics of action flying kick for martial arts athletes can be profile by Fig. (1).

Test equipment used in the experimental process are Footscan USB Plate plantar pressure plate system, a laptop computer, a camera, a tripod, height gauge, the scales, tape, etc. Test indicators are plantar pressure, peak pressure, the pressure, the pressure peak value, pulse, center of gravity height. The experimental steps including the following steps (Shown Fig (2)).

First, on plantar pressure distribution measurement system connection and debugging, set up the camera, two cameras to $90^{\circ}$, is located in the side of the athletes and positive, camera frequency 60 frames, the primary optical axis from the ground $1.1 \mathrm{~m}$.

Second, the object of study wear the same style, material of thin bottom rubber shoes, and test the research object's height and weight. 


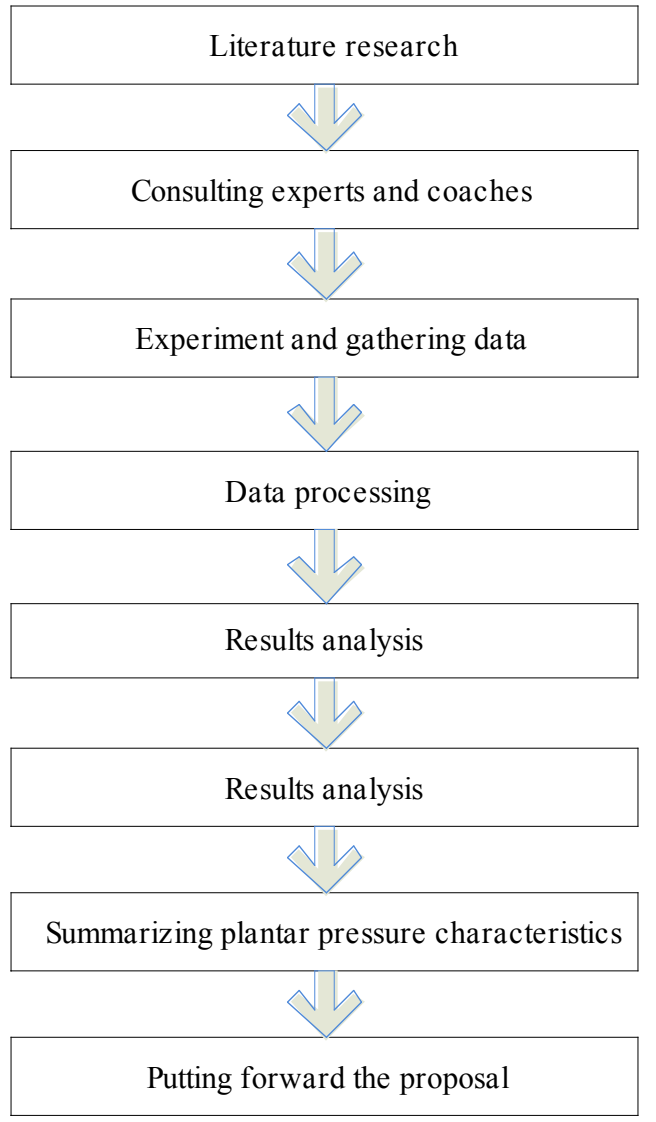

Fig. (1). The whole process of analysis.

Third, the research object to warm-up, open the machine equipment type, told the subjects of the experiment requirements, process, content, points for attention, and practice a set effective area.

Fourth, An official test, sampling each of the research object in the flying kick movement jumping the plantar pressure distribution of the data, synchronous photography camera, the object of study all adopt the right foot.

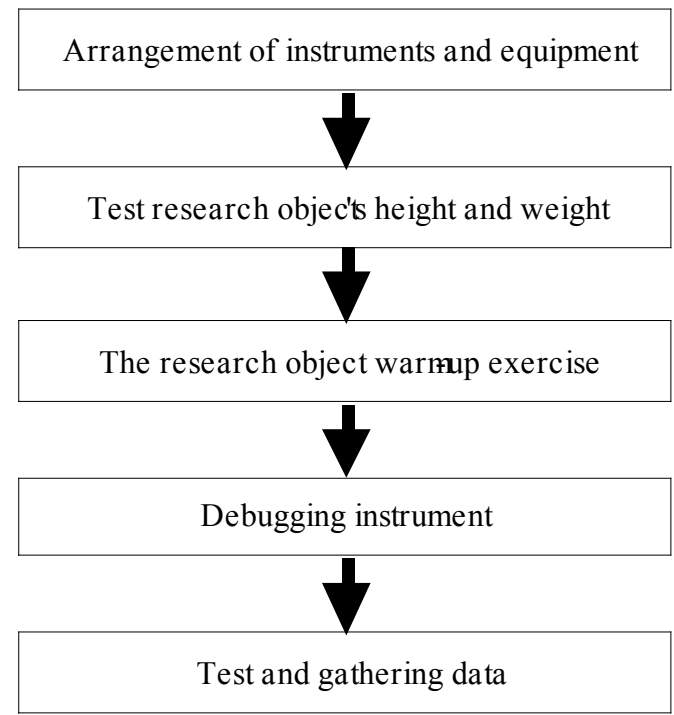

Fig. (2). The experimental steps.

\section{THE EXPERIMENT RESULTS ANALYSIS}

According to a foot anatomy, the plantar is divided into front feet, the arch and heel three parts. In order to facilitate research, the plantar will be divided into heel two areas(HL and $\mathrm{HM})$, the $\operatorname{arch}(\mathrm{MF})$, the first head of metatarsal bone(M1), the second head of metatarsal bone(M2), the third head of metatarsal bone(M3),the fourth head of metatarsal bone(M4), the fifth head of metatarsal bone(M5), the first phalanx(T1), and the second to fifth phalanx(T2 to T5), a total of ten regions.

For the experimental results, this paper mainly discussed the relationship between the plantar pressure and time and the relationship between pressure and time two aspects, and then analyze and summarize characteristics.

\subsection{Analysis of Characteristics of Plantar Pressure and Time}

Figs. (4) and (5) are foot-pressure curve over time when No. 1 and No. 2 martial arts athletes in Table 1 complete the action flying kick, where time is measured in milliseconds, the color of curves in the diagram in Fig. (3) correspond to the soles of the color of each partition.

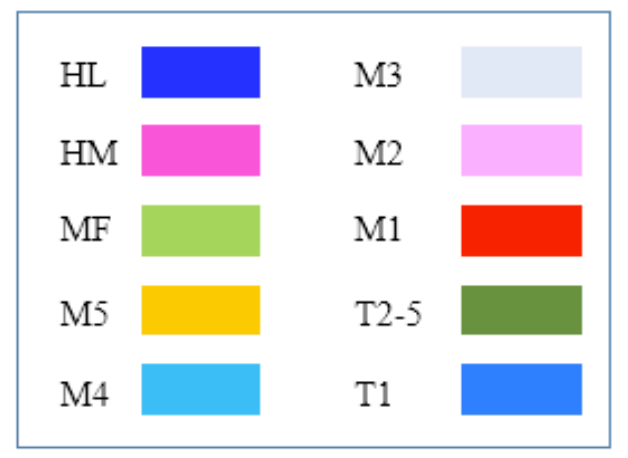

Fig. (3). Foot zoning map.

From the testing results known in the initial stage of jumping by the heel (HL and HM) first touchdown. And No.3 and No.6, MF, M5, M4 area during this time all the pressure, thus in the beginning of the jumping of No.3 and No.6 foot touchdown area than other subjects, when jumping action is better than other research object is stable, and it is not easy to damage. Look at the whole figure four to nine heel and T1 to T5, M1 to M5, MF is bimodal pattern in HM, maximum force basic HL is a foot rest began to rapidly rising, and when a foot got the maximum force moment HM, HL has less than $20 \mathrm{n}$, and even tend to zero, which can determine HM, the influence of the pressure for step pulse of HL parts of the very small, so, in the initial stage No.3 and No.6, jumping movement is good. The start jumping HM, land area of HL as far as possible big, in does not affect the premise of MF jumping part should also land.

Figs. (4) and (5) in the vertical lines represent a foot together biggest moment, the max force and max force moment of the six martial arts athletes in Table $\mathbf{1}$ are shown in Table 2 . 


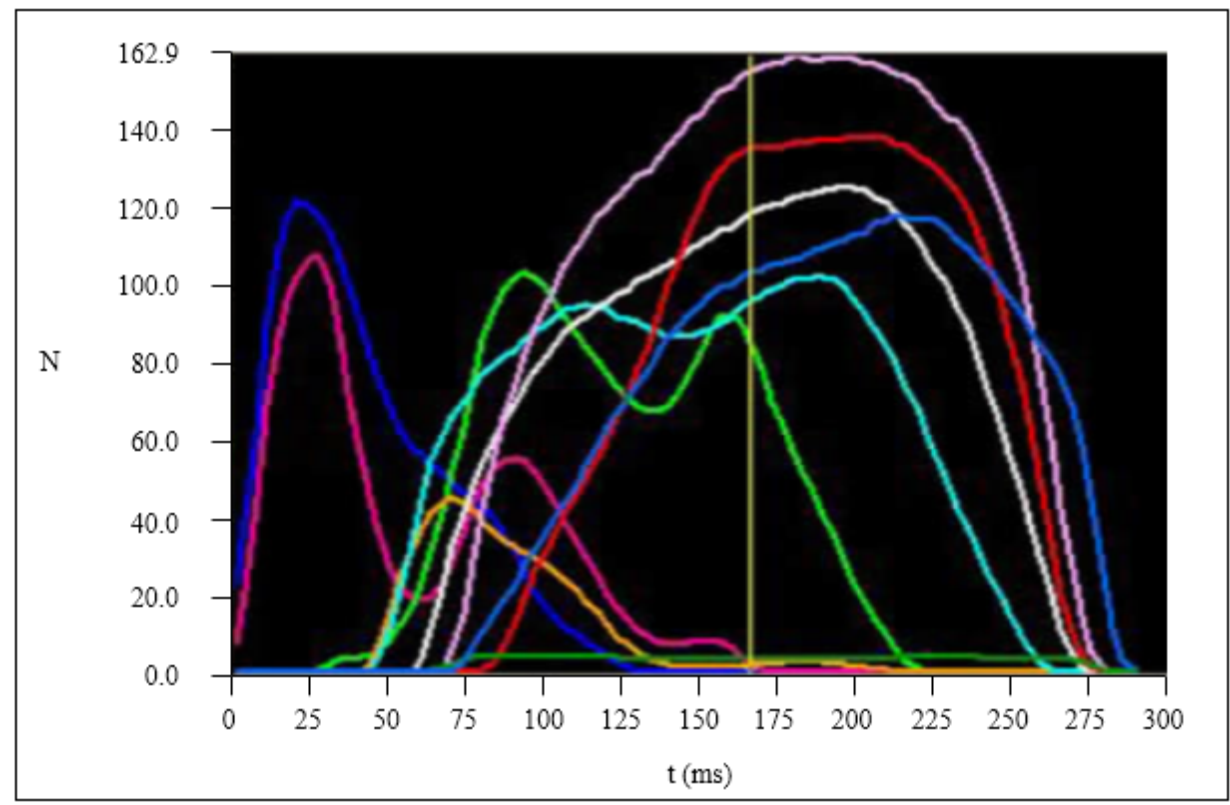

Fig. (4). Foot-pressure curve over time for no.1.

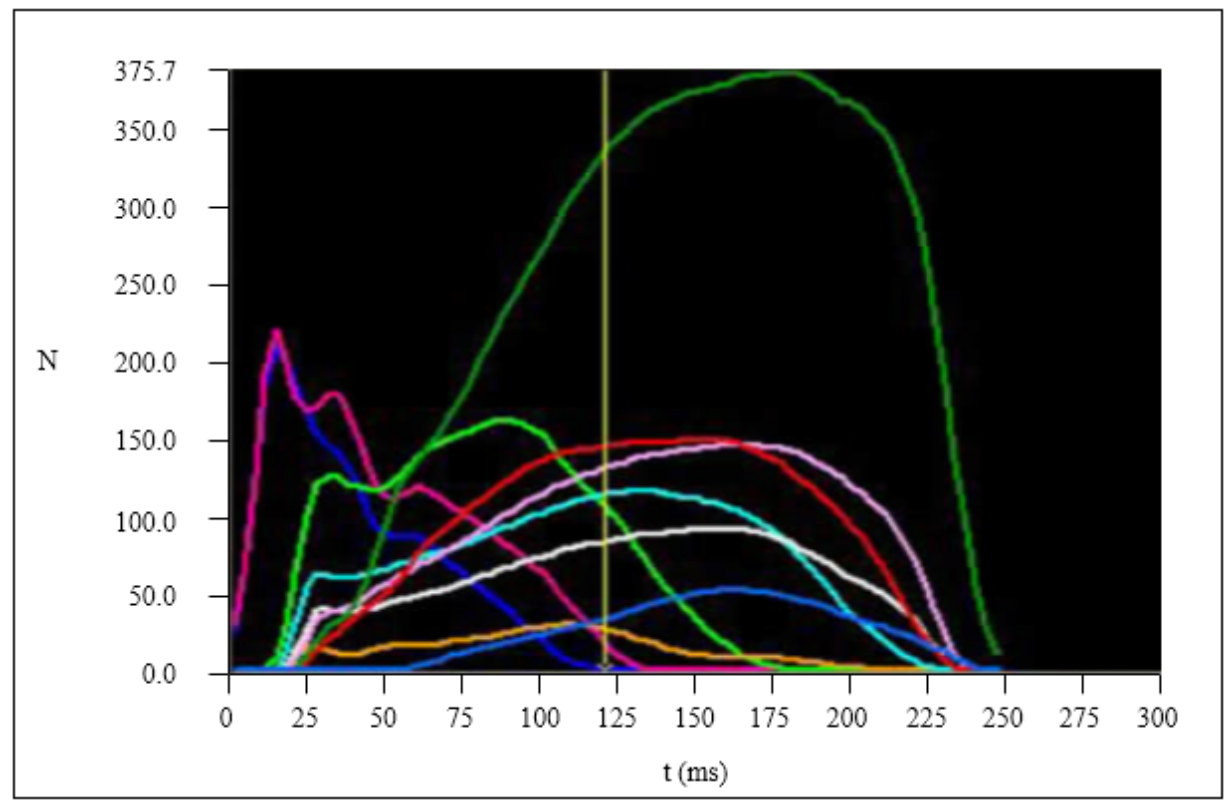

Fig. (5). Foot-pressure curve over time for no.2.

Table 2. The max force and max force moment of the six martial arts athletes.

\begin{tabular}{|c|c|c|c|c|c|}
\hline No. & Weight(kg) & Height(cm) & Max Force(N) & Max Time & 167 \\
\hline \hline 1 & 50 & 165 & 716 & 120 & 7.52 \\
\hline 2 & 55 & 165 & 963 & 93 & 143 \\
\hline 3 & 55 & 170 & 904 & 906 & 123 \\
\hline 4 & 60 & 172 & 684 & 107 & 4.16 \\
\hline 5 & 49 & 163 & 996 & 6.8 \\
\hline
\end{tabular}


The Table 2 shows that when the research object reach to the biggest force moment, the vertical acceleration of No.2 is largest, then respectively are No.6, No.3, No.4, No.1 and No.5. Human body not particle, however, under the condition of without considering other factors, it can be seen the jumping way and sports ability of No.2 is best, then respectively are No.6, No.3, No.4, No.1 and No.5.

\subsection{Analysis of Characteristics of Pressure and Time}

Figs. (6) and (7) are pressure curve over time when No.1 and No. 2 martial arts athletes in Table 1 complete the action flying kick, where time is measured in milliseconds. The research object can bear the biggest pressure and the area are shown in Table $\mathbf{3}$.

It can be seen from the result of characteristics of pressure and time that research object bear maximum pressure parts, respectively is M3 for No.1, M4 for No.2, M3 for No.3, M2 for No.4, M4 for No.5, M3 and M4 for No.6. In addition to the maximum pressure parts, the M2, M3 and T1 of No.1, the M2, M3 and T2 to T5 of No.2, the M2, M4 and T1 of No.3, the M3 of No.4, the M3 and T2 to T5 of No.1, the M2, M3 and T1 of No.1 also bear a lot of pressure. This shows fully convincingly that flying kick of empty jumping action by the stress mainly concentrated in the front feet, especially the sole part by stress, should practice to these areas to strengthen, to enhance the capacity of jumping, buffer a foot shock.

Table 3. The research object can bear the biggest pressure and the area.

\begin{tabular}{|c|c|c|}
\hline No. & Max Pressure(N/cm $\left.{ }^{2}\right)$ & Foot Zoning \\
\hline \hline 1 & $8.45 \pm 3.82$ & M3 \\
\hline 2 & $9.48 \pm 3.86$ & M4 \\
\hline 3 & $7.67 \pm 4.04$ & M3 \\
\hline 4 & $8.06 \pm 3.69$ & M2 \\
\hline 5 & $6.99 \pm 2.63$ & M4 \\
\hline 6 & $7.91 \pm 2.85$ & M3 and M4 \\
\hline
\end{tabular}

In the position of the peak pressure is the biggest burden load position, is also the most prone to damage location. The location of the maximum respectively are M3 for No.1 and

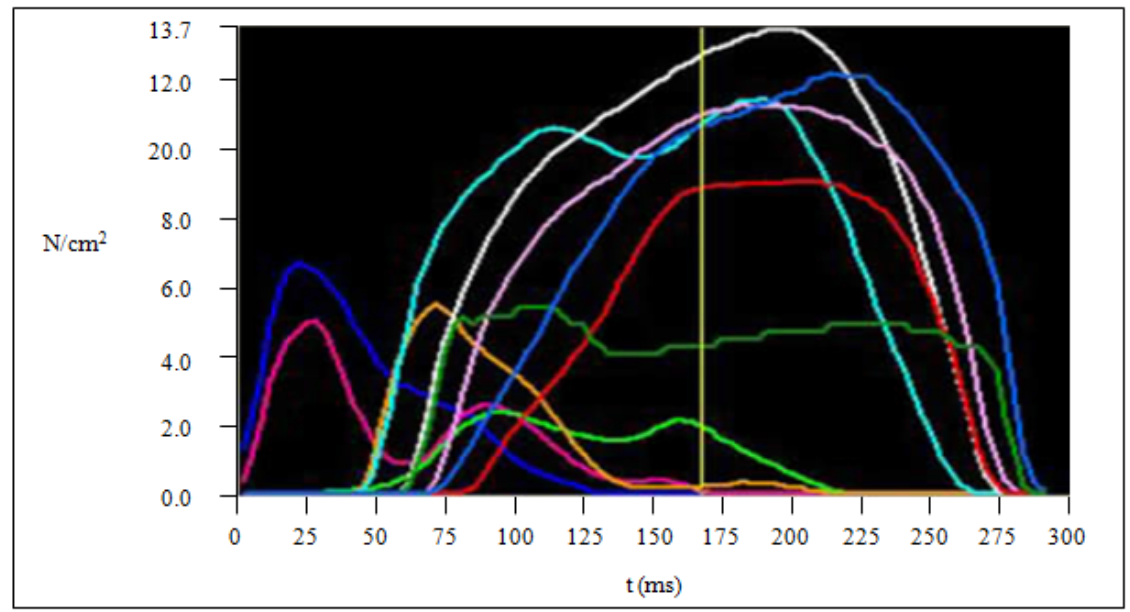

Fig. (6). Pressure curve over time for no.1.

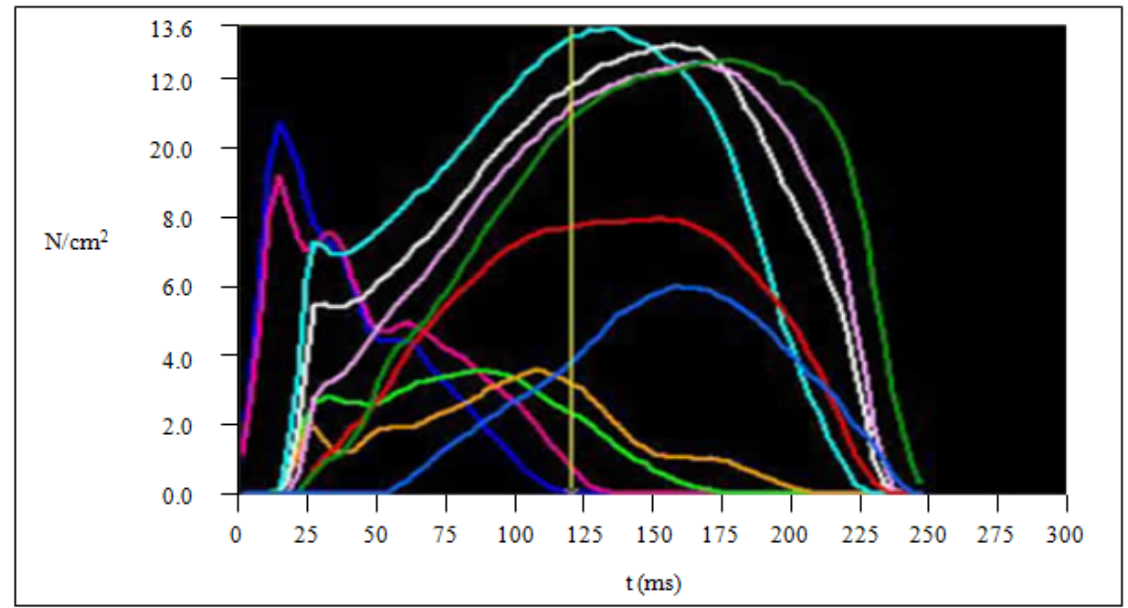

Fig. (7). Pressure curve over time for no.2. 
No.3, M4 for No.2, No5, and No.6, M2 for No.4. The position of the maximum for No.2, No.5 and No.6 is M4. That is the position of the fourth metatarsal is injury prone position.

\section{CONCLUSION}

First of all, this paper discussed the methods and steps for the analysis of the plantar pressure characteristics of action flying kick for martial arts athletes, including the research object, research methods and the experimental steps, summing up the process of the plantar pressure analysis and the experimental process. Then, the paper given six Jilin province martial arts athletes flying kick foot jump for bottom pressure test of the experimental results, and analyzed the experimental data in detail.

By analyzing the experimental results, it can be obtained some conclusion. Flying kick will begin with a heel brake pedal to beat, in the process of braking in different research object of braking phase. HM and HL area stress difference will lead to center of gravity be unsteady, even damage. It suggestion adjustment technique, HM and HL forcing equilibrium is advisable. Heel for empty jumping is very small, the influence of braking when under the premise of not affect empty jumping heel contact with the ground area should be as large as possible. Under maximum load in the process of jumping is M2, M3, M4, maximum peak pressure concentrated in M2, M3, M4, these three parts are in the ball, so the ball is under the load is also the biggest, strengthen the protection of this area can effectively prevent the occurrence of injury, improve the quality of the action.

\section{CONFLICT OF INTEREST}

The author confirms that this article content has no conflict of interest.

\section{ACKNOWLEDGEMENTS}

This work is supported by the Hunan Province Philosophy Social Science Foundation "Confusion and value orientation of face students sports competition" (NO: 12YBB124).

\section{PATIENT'S CONSENT}

Declared none.

\section{REFERENCES}

[1] T. Chaut, "A review of analytical techniques for gait data. Part I: Fuzzy, statistical and fractal methods," Gait and Posture, vol. 13, no. 1, pp. 49-66, 2001

[2] Y. Chen, and G. Yu, "F - Scan plantar pressure gait analyzer clinical application situation," Medical Bone Science Booklet, vol. 26, no. 3, pp. 187-189, 2005.

[3] J. M. Burnfield, C. D. Few, O. S. Mohamed, and J. Perry, "The influence of walking speed and footwear on plantar pressures in older adults," Clinical Biomechanics, vol. 19, no. 1, pp. 78-84, 2004.

[4] P. E. Martin, and R. C. Nelson, "The effect of carried load on the walking patterns of men and women," Ergonomics, vol. 29, no. 10, pp. 1191-1202, 1986.

[5] S. C. Wearing, S. R. Urry, and J. E. Smeathers, "Ground reaction forces at discrete sites of the foot derived from pressure plate measurements," Foot \& Ankle International, vol. 22, no. 8, pp. 653-661, 2001.

[6] E. Eils, S. Nolte, M. Tewes, L. Thorwesten, K. Völker, and D. Rosenbaum, "Modified pressure distribution patterns in walking following reduction plantar sensation," Journal of Biomechanics, vol. 35 , no. 10, pp. 1307-1313, 2002.

[7] L. Wang, and J. Li, "Application and development of foot pressure measurement technology," Zhejiang Sport Science, vol. 26, no. 1, pp. 40-43, 2004.

[8] M. Horvath, T. Tekla, and T. Jozsef, "Kinematic and kinetic analyses of Gait patterns in hemiplegic patients," Physical Education and Sport, vol. 1, no. 8, pp. 25-35, 2001.

[9] M. Josephine, and H. Mitul, "Foot pressure distribution during walking in young and old adults," BMC Geriatrics, vol. 19, no. 3, pp. 5-8, 2005.

[10] T. S. Gross, and R. P. Bunch, "Discrete normal plantar stress variations with running speed," Journal of Biomechanics, vol. 22, no. 6 , pp. 699-703, 1989

[11] N. Liu, H. Wang, and T. Li, "The plantar pressure distribution characteristics of action flying kick for wushu athletes," Journal of Captial University of Physical Education and Sports, vol. 25, no. 1, pp. 93-96, 2013.

[12] H. Sato, H. Sako, H. Mukae, A. Sata, and T. Takahashi, "Gait patterns of young Japanese woman," Journal of Human Ergology, vol. 20 , no. 1 , pp. $85-88,1991$

[13] J.A. Stebbins, M.E. Harrington, C. Giacomozzi, N. Thompson, A. Zavatsky, and T.N. Theologis, "Assessment of subdivision of plantar pressure measurement in children," Gait and Posture, vol. 17, no. 22 , pp. 372-376, 2005.

[14] E. Morag, and P. R. Cavanagh, "Structural and functional predictors of regional peak pressures under the foot during walking," Journal of Biomechanics, vol. 32, no. 4, pp. 359-370, 1999.

[15] J. Li, and L. Wang, "The application and progress of plantar pressure measurement technology in biomechanics research," Journal of Beijing University of Physical Education, vol. 28, no. 2, pp. 191193, 2005.

\begin{tabular}{lcc}
\hline Received: May 26, 2015 & Revised: July 14, 2015 & Accepted: August 10, 2015 \\
(C) Jin Fei Tan; Licensee Bentham Open. &
\end{tabular}

This is an open access article licensed under the terms of the Creative Commons Attribution Non-Commercial License (http://creativecommons.org/licenses/by-nc/3.0/) which permits unrestricted, non-commercial use, distribution and reproduction in any medium, provided the work is properly cited. 